\title{
Article
}

\section{Caught in the act: direct detection of Galactic Bars in the buckling phase}

\author{
Erwin, Peter and Debattista, Victor P \\ Available at https://clok.uclan.ac.uk/16808/ \\ Erwin, Peter and Debattista, Victor P orcid iconORCID: 0000-0001-7902-0116 \\ (2016) Caught in the act: direct detection of Galactic Bars in the buckling \\ phase. The Astrophysical Journal Letters, 825 (2). L30. ISSN 2041-8205
}

It is advisable to refer to the publisher's version if you intend to cite from the work. http://dx.doi.org/10.3847/2041-8205/825/2/L30

For more information about UCLan's research in this area go to http://www.uclan.ac.uk/researchgroups/ and search for < name of research Group>.

For information about Research generally at UCLan please go to http://www.uclan.ac.uk/research/

All outputs in CLoK are protected by Intellectual Property Rights law, including Copyright law. Copyright, IPR and Moral Rights for the works on this site are retained by the individual authors and/or other copyright owners. Terms and conditions for use of this material are defined in the policies page.

\section{CLoK}

Central Lancashire online Knowledge www.clok.uclan.ac.uk

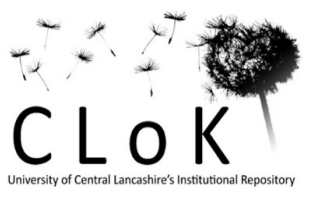




\title{
CAUGHT IN THE ACT: DIRECT DETECTION OF GALACTIC BARS IN THE BUCKLING PHASE
}

\author{
Peter Erwin ${ }^{1,2}$ and Victor P. Debattista ${ }^{3}$ \\ ${ }^{1}$ Max-Planck-Institut für extraterrestrische Physik, Giessenbachstrasse, D-85748 Garching, Germany; erwin@mpe.mpg.de \\ ${ }^{2}$ Universitäts-Sternwarte München, Scheinerstrasse 1, D-81679 München, Germany \\ ${ }^{3}$ Jeremiah Horrocks Institute, University of Central Lancashire, Preston PR1 2HE, UK \\ Received 2016 February 27; revised 2016 June 2; accepted 2016 June 12; published 2016 July 11
}

\begin{abstract}
The majority of massive disk galaxies, including our own, have stellar bars with vertically thick inner region, known as "boxy/peanut-shaped" (B/P) bulges. The most commonly suggested mechanism for the formation of $\mathrm{B} / \mathrm{P}$ bulges is a violent vertical "buckling" instability in the bar, something that has been seen in $N$-body simulations for over 20 years, but never identified in real galaxies. Here, we present the first direct observational evidence for ongoing buckling in two nearby galaxies (NGC 3227 and NGC 4569), including characteristic asymmetric isophotes and (in NGC 4569) stellar kinematic asymmetries that match buckling in simulations. This confirms that the buckling instability takes place and produces $\mathrm{B} / \mathrm{P}$ bulges in real galaxies. A toy model of bar evolution yields a local fraction of buckling bars consistent with observations if the buckling phase lasts $\sim 0.5-1$ Gyr, in agreement with simulations.
\end{abstract}

Key words: galaxies: bulges - galaxies: evolution - galaxies: spiral - galaxies: structure

\section{INTRODUCTION}

Approximately $60 \%-70 \%$ of disk galaxies in the local universe have stellar bars (e.g., Eskridge et al. 2000; Menéndez-Delmestre et al. 2007). A wide variety of observational evidence indicates that many bars are vertically thickened in their inner regions, appearing as "boxy" or "peanut-shaped" $(\mathrm{B} / \mathrm{P})$ bulges when seen edge-on; this includes our own Galaxy, whose bulge is mostly-if not entirely-part of its bar (e.g., Shen et al. 2010; Di Matteo et al. 2014). Edge-on galaxies with $\mathrm{B} / \mathrm{P}$ bulges show gas and stellar kinematics consistent with a rotating bar in the disk plane (Kuijken \& Merrifield 1995; Bureau \& Freeman 1999; Merrifield \& Kuijken 1999; Veilleux et al. 1999; Chung \& Bureau 2004); moderately inclined barred galaxies show isophotes consistent with the projection of $\mathrm{B} / \mathrm{P}$ bulges within the bars (Bettoni \& Galletta 1994; Quillen et al. 1997; Athanassoula \& Beaton 2006; Erwin \& Debattista 2013); and face-on barred galaxies show kinematic and morphological signatures of $\mathrm{B} / \mathrm{P}$ bulges as well (Méndez-Abreu et al. 2008; Laurikainen et al. 2014).

Recent studies suggest that failing to account for the presence of $\mathrm{B} / \mathrm{P}$ bulges can lead to significantly overestimating the luminosities and masses of "classical" (spheroidal) bulges in disk galaxies (Laurikainen et al. 2014; Athanassoula et al. 2015). This can potentially bias our understanding of how bulges are related to other galaxy properties, including the key correlations between supermassive black holes and bulges (e.g., Kormendy \& Ho 2013). Understanding the formation of $\mathrm{B} / \mathrm{P}$ bulges is thus an important part of understanding and constraining models of galaxy (and black hole) evolution.

The most frequently invoked mechanism for forming these structures is the buckling instability of the bar, a brief but violent vertical instability which occurs (in simulations) not long after the bar forms. In simulations, the formation of the bar increases the radial velocity dispersion of stars in the disk; this leads to a highly anisotropic velocity dispersion tensor and the vertical destabilization of the bar (Raha et al. 1991; Merritt \& Sellwood 1994). Following a phase of asymmetric vertical buckling, the inner part of the bar settles into the more vertically symmetric form of a $\mathrm{B} / \mathrm{P}$ bulge.

Despite over 20 years of simulations that show buckling, no observed buckling has yet been reported for real galaxies, a situation called "puzzling" in the review by Shen \& Li (2016). An alternative model proposes that $\mathrm{B} / \mathrm{P}$ bulges form by the trapping of single orbits into vertical resonances, leading to more gradual, vertically symmetric growth (Combes et al. 1990; Quillen 2002; Debattista et al. 2006; Berentzen et al. 2007; Quillen et al. 2014). In some simulations, the presence of gas weakens or prevents buckling, while still allowing symmetric bar thickening (Berentzen et al. 1998; Debattista et al. 2006; Berentzen et al. 2007; Wozniak \& Michel-Dansac 2009). Thus, it is not clear that real galaxies must suffer the buckling instability. There are also no clear, strong differences due to the different formation mechanisms in the resulting end-stage $\mathrm{B} / \mathrm{P}$ bulges, making it difficult to determine from observations of existing $\mathrm{B} / \mathrm{P}$ bulges how they were formed.

In this Letter, we present evidence for ongoing buckling in the bars of two local spiral galaxies (NGC 3227 and NGC 4569), thus demonstrating that buckling of bars definitely occurs in real galaxies. We also argue that the observed fraction of buckling bars at $z=0$ is at least broadly consistent with most (or even all) $\mathrm{B} / \mathrm{P}$ bulges being the result of buckling, if the buckling phase lasts $\sim 0.5-1 \mathrm{Gyr}$ - as is predicted by $N$-body simulations.

\section{BUCKLING AND MORPHOLOGY}

\subsection{N-body Simulations}

$N$-body simulations have long predicted that bars should buckle some time after they form. In this Letter, we use four such simulations, three of which were previously analyzed in Erwin \& Debattista (2013). For consistency, we use a similar naming scheme: "simulation A-C" in this paper correspond to "runs A-C" in Erwin \& Debattista. These three simulations are described in more detail in Erwin \& Debattista and (for simulations B and C) in Sellwood \& Debattista (2009); they included 300,000-500,000 stellar particles in the disk and softening lengths of $60 \mathrm{pc}$ (simulation $\mathrm{A}$ ) or 0.05 natural units (B 



Figure 1. Edge-on and inclined views of $N$-body simulations before, during, and after vertical buckling. Panels (a)-(c) show log-scaled isodensity contours of simulation $\mathrm{C}$ for edge-on (upper sub-panels, with bar perpendicular to line of sight) and inclined views (lower sub-panels, $i=60^{\circ}$, bar oriented $30^{\circ}$ from line of nodes before inclining galaxy). (a) Before buckling, showing the symmetric, vertically thin bar. (b) During buckling: vertical asymmetry (upper sub-panel) translates to an asymmetric, trapezoidal inner region in the lower sub-panel (red contours) and outer-bar "spurs" (green), offset in same direction (arrows) from the major axis of the inner region (cyan line) in the lower sub-panel. (c) After buckling: the symmetric boxy/peanut-shaped (B/P) bulge projects to rectangular inner contours (red) and counter-offset spurs (green contours, arrows); the projected bar now has $180^{\circ}$ rotational symmetry about the galaxy center. The small inset panels show cartoon versions of the basic buckling and post-buckling projected morphologies (red trapezoid/box + green spurs). (d)-(f) As for lower sub-panel of (b), but now showing simulation $\mathrm{C}$ later in the buckling process (d) and simulations B and D during their buckling phases (e) and (f); all three are seen with $i=65^{\circ}$.

and C). Simulation D is almost identical to model T2 of Debattista et al. (2016). It is comprised of thin+thick disks inside a dark matter halo, with three million stellar particles and a softening length of $50 \mathrm{pc}$. The two disks have roughly the same radial velocity dispersion profile, but the thin disk has half the height of the thick disk (versus a height ratio of one-fourth for model T2); see Debattista et al. (2016) for further details.

\subsection{Projected Morphology}

Erwin \& Debattista (2013) compared $N$-body simulations and real barred galaxies to show that it was possible to identify $\mathrm{B} / \mathrm{P}$ bulges in bars even at inclinations as low as $\sim 40^{\circ}$. (The advantage of intermediate inclinations-e.g., $i \lesssim 70^{\circ}$ - over more edge-on orientations is that the bar as a whole is readily identifiable and measurable, regardless of whether its interior is 

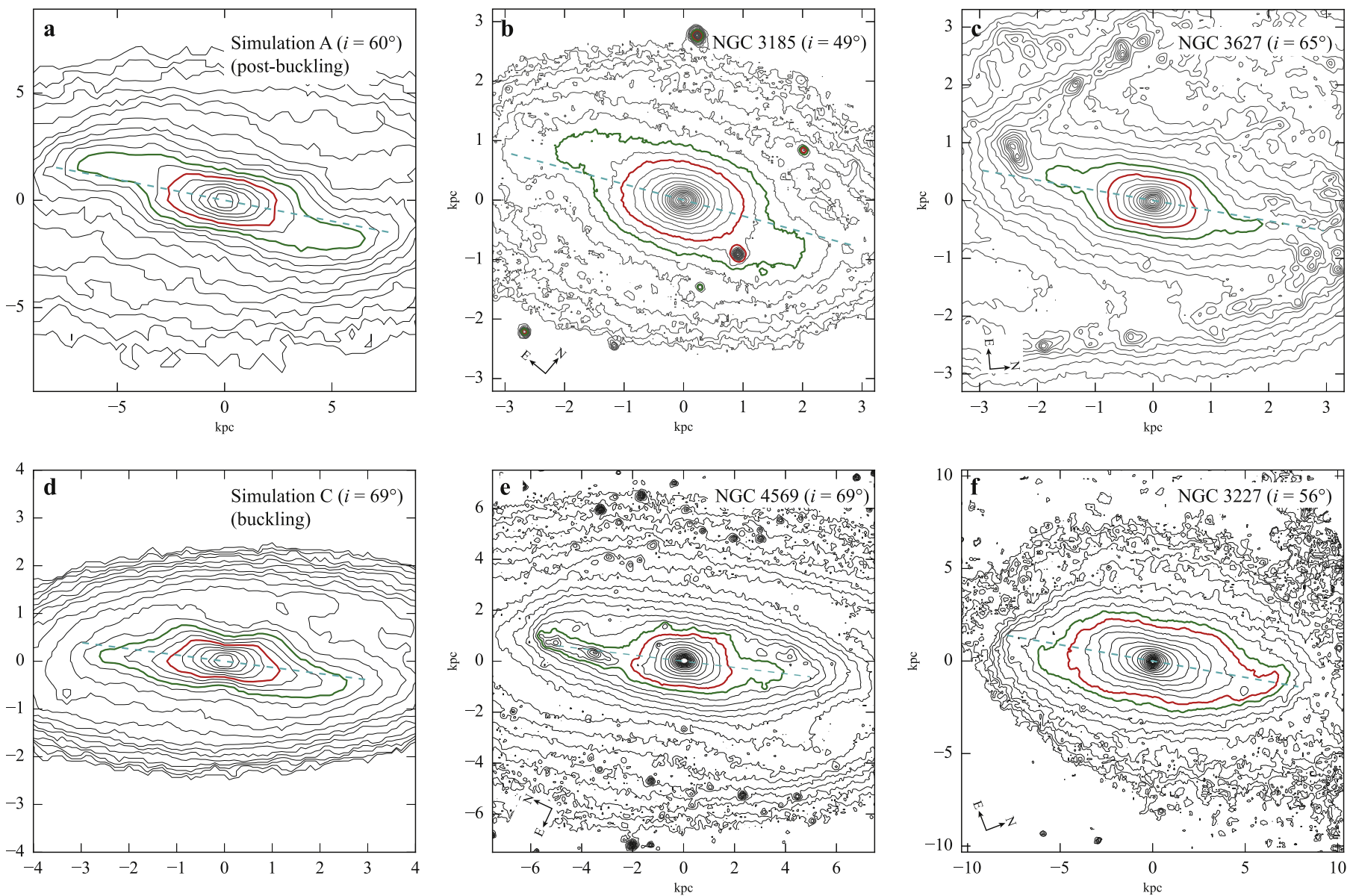

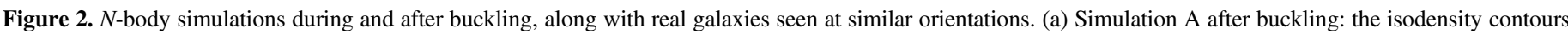

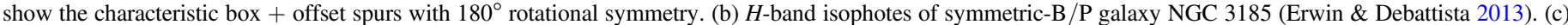

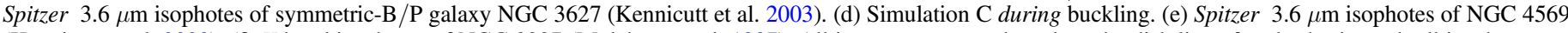

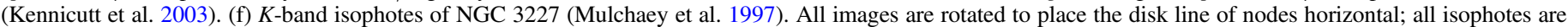

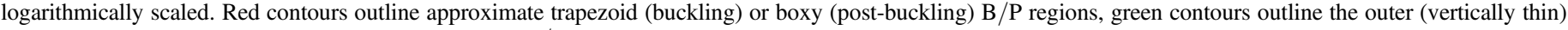
bar spurs, and the dashed cyan lines show the trapezoid/box major axes.

vertically thick.) The key isophotal signatures are a broad, often boxy inner zone (corresponding to the vertically thick, B/P part of the bar) and thinner "spurs" extending beyond (corresponding to the vertically thin outer parts of the bar); the spurs are usually offset from the major axis of the inner zone so that the projected bar has $180^{\circ}$ rotational symmetry on the sky (Figures 1(c) and 2(a)).

When simulations in the buckling phase are viewed at the same intermediate inclinations, however, the projected bar shows trapezoidal inner isophotes (corresponding to the main buckling region) and outer spurs which are offset in the same direction relative to the bar's observed major axis, forming a continuation of the trapezoid's long side (Figures 1(b), (d)-(f) and $2(\mathrm{~d})$ ). This morphology clearly differs from the symmetricbox plus counter-offset spurs seen in post-buckling bars with their symmetric $\mathrm{B} / \mathrm{P}$ bulges.

\section{DETECTION OF BUCKLING BARS IN NGC 3227 AND NGC 4569}

\subsection{Morphology}

As part of a survey of local barred galaxies with favorable orientations for detecting and measuring projected $\mathrm{B} / \mathrm{P}$ bulges (P. Erwin \& V. P. Debattista 2016, in preparation), we found two examples of bars with morphologies indicating that they are currently in the buckling phase: NGC 3227 and NGC 4569 (M90). In both galaxies, the inner or middle region of the bar shows quasi-trapezoidal isophotes (most clearly in NGC 4569), while the outer-bar spurs are offset in the same direction and connect to the long side of the trapezoid (Figures 2(e) and (f)). This morphology is a good match to the general appearance of projected $N$-body bars in the buckling phase. Although both galaxies are currently experiencing star formation within their bars, comparison of their near-infrared morphologies with published $\mathrm{H} \alpha$ images allows us to rule out the possibility that recent star formation is responsible for the overall morphology (Figure 3).

The best case is probably NGC 4569, which shows a symmetric inner trapezoid and clear offset spurs; it differs from the simulations (Figure 1) in having a relatively compact buckling region, with a half-length $R_{\text {box }} \sim 1.9 \mathrm{kpc}$ (measured along its long axis), quite small relative to the bar as a whole $\left(R_{\mathrm{box}} / L_{\mathrm{bar}} \approx 0.29\right.$, where $L_{\mathrm{bar}}=$ half-length of the bar). The trapezoidal region in NGC 3227 is much larger $\left(R_{\mathrm{box}}=4.7 \mathrm{kpc}, R_{\mathrm{box}} / L_{\mathrm{bar}}=0.54\right)$, with some resemblance to simulation B (Figure 1(e)); the interior of the trapezoid appears less symmetric, which may be partly due to emission from recent star formation (Figure 3). Nonetheless, the outer 

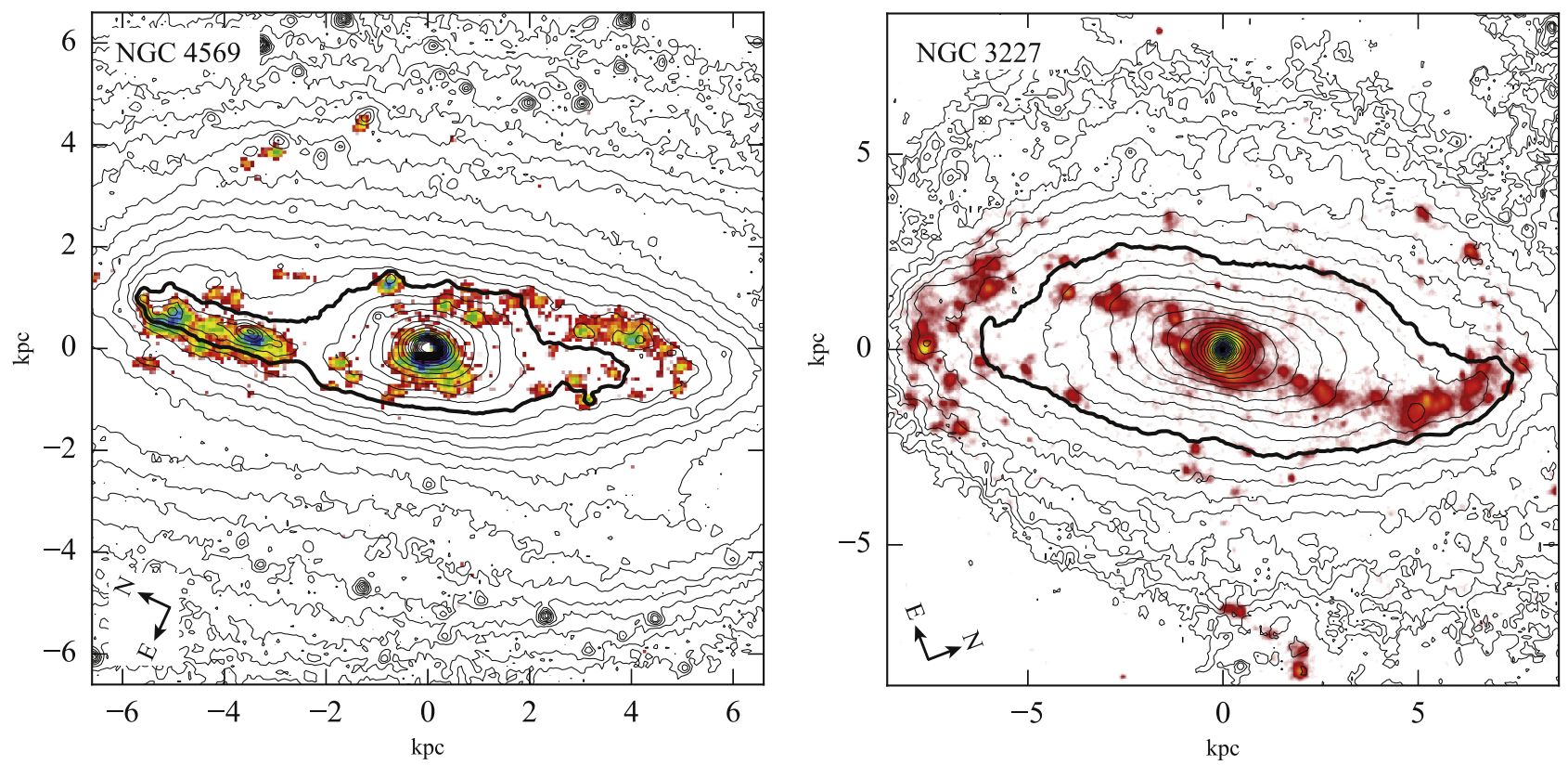

Figure 3. Star formation and near-IR morphology for galaxies with buckling bars. We show logarithmically scaled near-IR isophotes (left black contours: Spitzer $3.6 \mu \mathrm{m}$ from Kennicutt et al. 2003; right black countours: $K$-band from Mulchaey et al. 1997) and H $\alpha$ emission (left color: Knapen et al. 2004; right color: Koopmann et al. 2001); thicker contours outline spurs in each bar. Galaxies are rotated to place disk major axes horizontal. Star formation enhances the northern spurs in each galaxy, but is unrelated to the southern spurs. All four spurs are thus primarily due to the underlying stellar structure of the bars, not recent star formation.

Table 1

Stellar Kinematic Data

\begin{tabular}{|c|c|c|c|c|c|c|c|}
\hline Galaxy & $\begin{array}{l}i \\
\left({ }^{\circ}\right)\end{array}$ & $\begin{array}{l}\text { PA } \\
\left({ }^{\circ}\right)\end{array}$ & $\begin{array}{c}\text { Bar PA } \\
\left(^{\circ}\right)\end{array}$ & $\begin{array}{c}\Delta \mathrm{PA} \\
\left(^{\circ}\right)\end{array}$ & $\begin{array}{c}\text { Slit PA } \\
\left(^{\circ}\right)\end{array}$ & $\begin{array}{c}R_{\mathrm{box}} \\
\left({ }^{\prime \prime}\right)\end{array}$ & Source \\
\hline NGC 615 & 69 & 158 & 162 & 11 & 155 & 11 & 1 \\
\hline NGC 1023 & 66 & 69 & 58 & 26 & 87 & 35 & 2 \\
\hline NGC 2962 & 53 & 7 & 168 & 30 & 175 & 17 & 3 \\
\hline NGC 3031 & 58 & 150 & 160 & 18 & 137 & 97 & 2 \\
\hline NGC 3627 & 65 & 175 & 161 & 31 & 161 & 18 & 4 \\
\hline NGC 4293 & 63 & 65 & 77 & 25 & 75 & 17 & 5 \\
\hline NGC 4429 & 63 & 90 & 106 & 32 & 93 & 45 & 6 \\
\hline NGC 4569 & 69 & 25 & 15 & 26 & 15 & 24 & 7 \\
\hline NGC 4725 & 42 & 40 & 50 & 13 & 35 & 63 & 8 \\
\hline NGC 6744 & 52 & 21 & 177 & 36 & 0 & 29 & 9 \\
\hline NGC 7531 & 59 & 22 & 7 & 27 & 15 & 14 & 1 \\
\hline
\end{tabular}

Note. For each galaxy we list its name, inclination, the position angle (on the sky) of the disk, the position angle of the bar, the deprojected angle between the bar and disk major axis, the position angle of the slit (or pseudo-slit for IFU data), the radius of the $\mathrm{B} / \mathrm{P}$ bulge $R_{\mathrm{box}}$ within the bar, and the sources of kinematic data used for Figure 4. References for kinematic data: (1) Pizzella et al. 2004; (2) Fabricius et al. 2012; (3) Simien \& Prugniel 2000; (4) Dumas et al. 2007; (5) Falcón-Barroso et al. 2006; (6) Simien \& Prugniel 1997; (7) Cortés et al. 2015; (8) Héraudeau et al. 1999; (9) Bettoni \& Galletta 1997.

isophotes show the characteristic offset-spurs pattern. The relative sizes of the buckling regions in these two galaxies fall near the lower and upper limits for the range of symmetric $\mathrm{B} / \mathrm{P}$ bulge sizes seen in local barred galaxies, $R_{\mathrm{box}} / L_{\mathrm{bar}}=0.26-$ 0.58 , with a mean of 0.38 (Erwin \& Debattista 2013).

\subsection{Stellar Kinematics}

The buckling phase in our simulations is also accompanied by asymmetries in the stellar velocity dispersion $\sigma$, measured along the major axis of the bar. For simulated bars before and after the buckling phase, the dispersion is roughly symmetric about the center, $\sigma(-x) / \sigma(+x) \approx 1$, where $x$ is measured along the bar major axis. During the buckling phase, however, the dispersion becomes strongly asymmetric. To quantify this, we computed a mean asymmetry measure:

$$
A_{\sigma}(R)=\left|1-\frac{1}{N} \sum_{i=1}^{N} \sigma\left(-x_{i}\right) / \sigma\left(+x_{i}\right)\right|,
$$

where the dispersion is measured in $N$ radial bins $x_{i}$ on either side of the galaxy center, out to a maximum distance $R=\left|x_{N}\right|$. Symmetric dispersion profiles have values of $A_{\sigma}$ close to 0 , while more asymmetric profiles have larger values.

To determine $A_{\sigma}$ for a simulation, we first matched the orientation of NGC 4569 (the only one of the two galaxies with sufficiently extended stellar kinematics) by rotating the simulation so that the bar was $\triangle \mathrm{PA}=26^{\circ}$ away from the line of nodes, and then inclining it by $69^{\circ}$ about the line of nodes. We measured velocity dispersions as the square root of the variance of particle line of sight velocities, using a slit width of 0.2 and evenly spaced bins of radial size 0.05 (simulation units) along the major axis of the projected simulation. For observational comparison, we used published velocity dispersion data for NGC 4569 and ten barred galaxies with known (symmetric) $\mathrm{B} / \mathrm{P}$ bulges which had inclinations and bar orientations similar to the two buckling-bar galaxies; these galaxies and the sources of kinematic data are listed in Table 1 . For all but two of the symmetric-B/P galaxies, we use long-slit data, if the position angle of the slit was within $30^{\circ}$ of the bar major axis. For NGC 3627 and NGC 4293, we extracted pseudo-long-slit measurements along the bar major axes from published integral-field-unit (IFU) velocity dispersion data (Falcón-Barroso et al. 2006; Dumas et al. 2007). Finally, we derived velocity dispersion measurements for NGC 4569 from the IFU data of Cortés et al. (2015). 

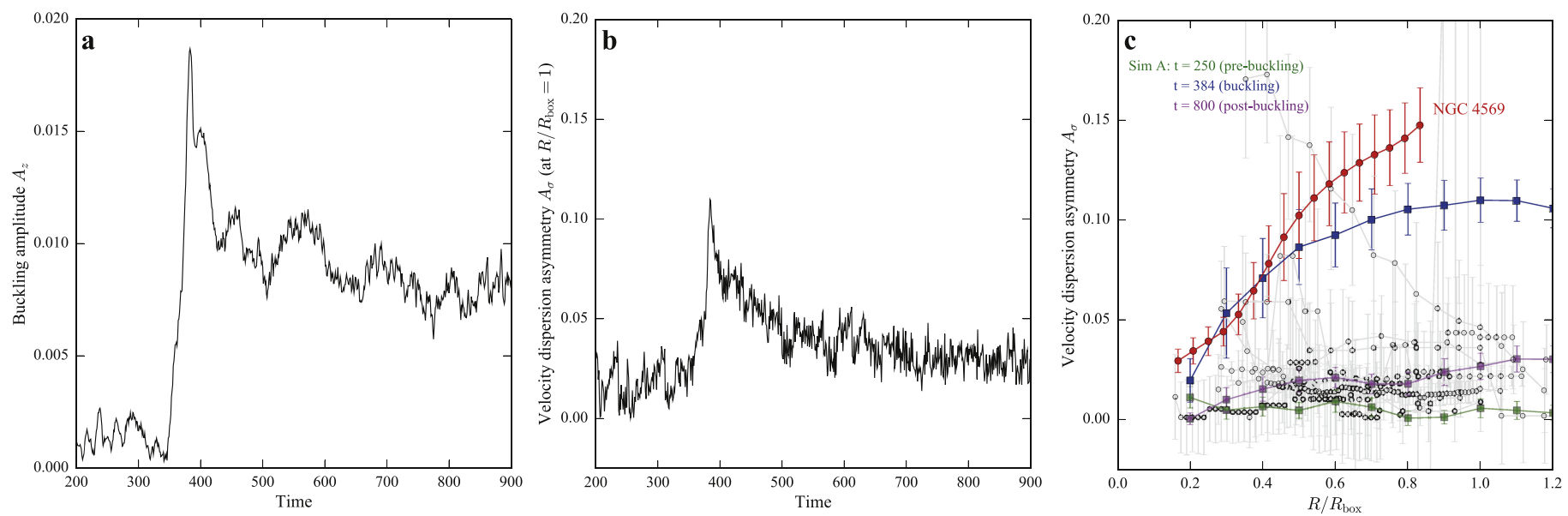

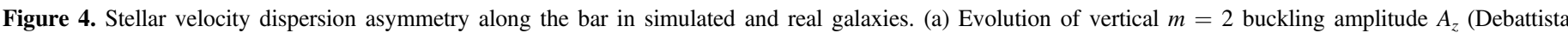

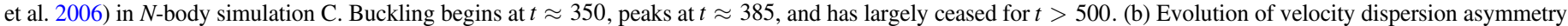

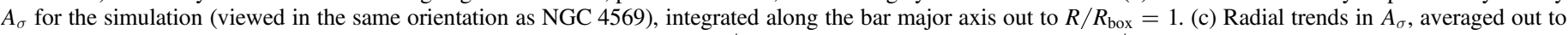

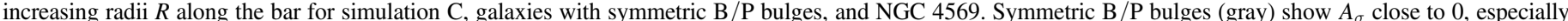

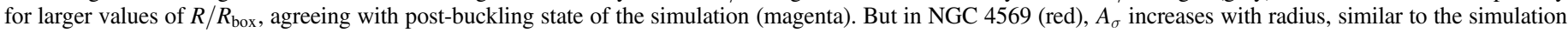
during buckling (blue). Error bars are standard deviations from 1000 rounds of bootstrap resampling.

Figure 4(b) shows the time evolution of $A_{\sigma}$ in simulation C, with $R$ equal to the size of the boxy/trapezoidal region once it has formed ( $R=R_{\mathrm{box}} \approx 1$ in simulation units). The onset of buckling at $t \approx 350$ (Figure 4(a)) is accompanied by rapid growth in the dispersion asymmetry (Figure 4(b)), which gradually returns to near symmetry $\left(A_{\sigma}<0.05\right)$ as the inner part of the post-buckled bar settles into a mature $\mathrm{B} / \mathrm{P}$ bulge. Figure 4(c) shows radial trends in $A_{\sigma}(R)$ for NGC 4569 and the ten symmetric $\mathrm{B} / \mathrm{P}$ galaxies, and also for representative times from simulation $\mathrm{C}$. Galaxies with symmetric B/P bulges (gray symbols) show minimal asymmetry (in almost all cases $A_{\sigma}<0.05$ ), especially for larger $R$, as do the pre- and postbuckling simulation profiles. For NGC 4569 (red symbols), however, the asymmetry increases as $R$ approaches the size of the buckling region-just as in the simulation during the buckling phase (blue symbols).

\section{THE EVOLUTION OF BARRED GALAXIES AND THE FREQUENCY OF BUCKLING}

We are currently studying a sample of local barred galaxies to identify and measure B/P bulges (P. Erwin \& V. P. Debattista 2016 , in preparation). This sample is diameter- and distancelimited $\left(D_{25} \leqslant 33^{\prime} 0\right.$ and $D \lesssim 30 \mathrm{Mpc}$, to ensure adequate resolution of bar interiors) and includes a total of 84 barred S0-Sd galaxies which have inclinations and orientations that maximize the detectability of $\mathrm{B} / \mathrm{P}$ bulges $\left(i=40^{\circ}-70^{\circ}\right.$, deprojected $\triangle \mathrm{PA}$ between bar and disk major axis $\left.<60^{\circ}\right)$. All galaxies were examined (using near-IR images) for the morphology of B/P bulges (Erwin \& Debattista 2013). We find $\mathrm{B} / \mathrm{P}$ bulges to be extremely common in high-stellar-mass galaxies: they are present in $\sim 80 \%$ of the 44 barred disks with $\log M_{\star} \gtrsim 10.4 M_{\odot}$.

The two buckling-bar galaxies were identified as part of this sample, and are in the high-stellar-mass $\left(\log M_{\star} \geqslant 10.4\right)$ subsample. The frequency of observed buckling in local, high-mass barred galaxies is thus $f_{\text {buck }}=4.5_{-2.3}^{+4.3} \%$. Is this frequency high or low? Put another way, is it consistent with the possibility that many-or even all $-\mathrm{B} / \mathrm{P}$ bulges are the result of the buckling instability?
To test this hypothesis, we adopted a toy galaxy-evolution model in which the fraction of disk galaxies with visible bars is a linear function of redshift, equal to $F_{0}$ at $z=0$ and decreasing to 0 at redshift $z_{i}$; we based this on the observed evolution of bar fraction with redshift in spiral galaxies (e.g., Sheth et al. 2008; Cameron et al. 2010; Melvin et al. 2014) and recent cosmologically motivated simulations of bar formation and growth (Kraljic et al. 2012). (We note that the simulated galaxies in Kraljic et al. (2012) all have $\log M_{\star}>10.2$, consistent with the local high-mass subsample we are considering, and that the high-redshift observational studies have similar lower limits on $M_{\star}$.) Following the pattern observed in $N$-body simulations, we assumed that there is a delay between bar formation and buckling, equal to $\Delta T_{\text {non-B/P }}$ Gyr, followed by a visible buckling phase lasting $\delta T_{\text {buck }}$ Gyr, and ending with a permanently $\mathrm{B} / \mathrm{P}$-hosting bar.

We performed a Markov Chain Monte Carlo analysis of this model, computing the likelihood as the product of individual binomial likelihoods for the $z=0$ fraction of galaxies with bars $f_{\text {bar }}$, the fraction of bars with $\mathrm{B} / \mathrm{P}$ bulges $f_{\mathrm{B} / \mathrm{P}}$, and the fraction of bars currently buckling $f_{\text {buck }}$, compared with the observed counts in our local high-mass subsample (P. Erwin \& V. P. Debattista 2016, in preparation); we assumed a flat prior for $0<z_{i}<2$ and flat priors for values $\geqslant 0$ for the other parameters. We used the emcee ensemble sampler code of Foreman-Mackey et al. (2013), with 50 separate chains and 500 steps per chain, discarding the first 150 steps in each chain. After marginalizing over $F_{0}$ and $z_{i}$ as nuisance parameters, we find $\Delta T_{\text {non }-\mathrm{B} / \mathrm{P}}=2.2_{-1.1}^{+1.3} \mathrm{Gyr}$ and $\delta T_{\text {buck }}=0.8_{-0.4}^{+0.7} \mathrm{Gyr}$ (medians and $68 \%$ confidence intervals). These are in good agreement with values from $N$-body simulations, which typically find $\Delta T_{\text {non }-\mathrm{B} / \mathrm{P}} \sim 1-2 \mathrm{Gyr}$ and $\delta T_{\text {buck }} \sim 0.5-1 \mathrm{Gyr}$ (Martinez-Valpuesta \& Shlosman 2004; Martinez-Valpuesta et al. 2006; Saha et al. 2013).

We conclude that the observed frequency of buckling bars at $z=0$ is consistent with the predictions of $N$-body simulations, and that the buckling instability could plausibly account for most if not all instances of $\mathrm{B} / \mathrm{P}$ bulges in massive disk galaxies in the local universe. (We note, however, that given the small numbers involved, our observations do not rule out the 
possibility that some of $\mathrm{B} / \mathrm{P}$ bulges could be the result of alternate, symmetric growth mechanisms.) Direct confirmation of this would involve imaging of bars in the buckling phase at higher redshifts. Our simple model predicts a maximum buckling fraction of $f_{\text {buck }} \sim 0.4$ at $z \sim 0.7$; near-IR detection of buckling in large bars $\left(L_{\mathrm{bar}} \gtrsim 4 \mathrm{kpc}\right)$ at this redshift is feasible with the upcoming James Webb Space Telescope.

We are pleased to thank Guillermo Blanc, Juan Cortés, Eric Emsellem, and Jésus Falcón-Barroso for generously making their kinematic data available; we also thank the referee (Juntai Shen), Jerry Sellwood, Roberto Saglia, Jairo Méndez-Abreu, and David Trinkle for useful comments on earlier drafts. V.P. D. was supported by STFC Consolidated grant \# ST/ M000877/1. V.P.D. also acknowledges with great pleasure the support of the Pauli Center for Theoretical Studies, which is supported by the Swiss National Science Foundation (SNF), the University of Zürich, and ETH Zürich.

This research made use of the NASA/IPAC Extragalactic Database (NED), which is operated by the Jet Propulsion Laboratory, California Institute of Technology, under contract with the National Aeronautics and Space Administration.

Facility: Spitzer (IRAC).

\section{REFERENCES}

Athanassoula, E., \& Beaton, R. L. 2006, MNRAS, 370, 1499

Athanassoula, E., Laurikainen, E., Salo, H., \& Bosma, A. 2015, MNRAS, 454,3843

Berentzen, I., Heller, C. H., Shlosman, I., \& Fricke, K. J. 1998, MNRAS, 300,49

Berentzen, I., Shlosman, I., Martinez-Valpuesta, I., \& Heller, C. H. 2007, ApJ, 666, 189

Bettoni, D., \& Galletta, G. 1994, A\&A, 281, 1

Bettoni, D., \& Galletta, G. 1997, A\&AS, 124, 61

Bureau, M., \& Freeman, K. C. 1999, AJ, 118, 126

Cameron, E., Carollo, C. M., Oesch, P., et al. 2010, MNRAS, 409, 346

Chung, A., \& Bureau, M. 2004, AJ, 127, 3192

Combes, F., Debbasch, F., Friedli, D., \& Pfenniger, D. 1990, A\&A, 233, 82

Cortés, J. R., Kenney, J. D. P., \& Hardy, E. 2015, ApJS, 216, 9

Debattista, V. P., Mayer, L., Carollo, C. M., et al. 2006, ApJ, 645, 209
Debattista, V. P., Ness, M., González, O. A., et al. 2016, MNRAS, submitted Di Matteo, P., Haywood, M., Gómez, A., et al. 2014, A\&A, 567, A122

Dumas, G., Mundell, C. G., Emsellem, E., \& Nagar, N. M. 2007, MNRAS, 379, 1249

Erwin, P., \& Debattista, V. P. 2013, MNRAS, 431, 3060

Eskridge, P. B., Frogel, J. A., Pogge, R. W., et al. 2000, AJ, 119, 536

Fabricius, M. H., Saglia, R. P., Fisher, D. B., et al. 2012, ApJ, 754, 67

Falcón-Barroso, J., Bacon, R., Bureau, M., et al. 2006, MNRAS, 369, 529

Foreman-Mackey, D., Hogg, D. W., Lang, D., \& Goodman, J. 2013, PASP, 125,306

Héraudeau, P., Simien, F., Maubon, G., \& Prugniel, P. 1999, A\&AS, 136, 509

Kennicutt, R. C., Jr., Armus, L., Bendo, G., et al. 2003, PASP, 115, 928

Knapen, J. H., Stedman, S., Bramich, D. M., Folkes, S. L., \& Bradley, T. R. 2004, A\&A, 426, 1135

Koopmann, R. A., Kenney, J. D. P., \& Young, J. 2001, ApJS, 135, 125

Kormendy, J., \& Ho, L. C. 2013, ARA\&A, 51, 511

Kraljic, K., Bournaud, F., \& Martig, M. 2012, ApJ, 757, 60

Kuijken, K., \& Merrifield, M. R. 1995, ApJL, 443, L13

Laurikainen, E., Salo, H., Athanassoula, E., Bosma, A., \& Herrera-Endoqui, M. 2014, MNRAS, 444, L80

Martinez-Valpuesta, I., \& Shlosman, I. 2004, ApJL, 613, L29

Martinez-Valpuesta, I., Shlosman, I., \& Heller, C. 2006, ApJ, 637, 214

Melvin, T., Masters, K., Lintott, C., et al. 2014, MNRAS, 438, 2882

Méndez-Abreu, J., Corsini, E. M., Debattista, V. P., et al. 2008, ApJL, 679, L73

Menéndez-Delmestre, K., Sheth, K., Schinnerer, E., Jarrett, T. H., \& Scoville, N. Z. 2007, ApJ, 657, 790

Merrifield, M. R., \& Kuijken, K. 1999, A\&A, 345, L47

Merritt, D., \& Sellwood, J. A. 1994, ApJ, 425, 551

Mulchaey, J. S., Regan, M. W., \& Kundu, A. 1997, ApJS, 110, 299

Pizzella, A., Corsini, E. M., Vega Beltrán, J. C., \& Bertola, F. 2004, A\&A, 424,447

Quillen, A. C. 2002, AJ, 124, 722

Quillen, A. C., Kuchinski, L. E., Frogel, J. A., \& Depoy, D. L. 1997, ApJ, 481,179

Quillen, A. C., Minchev, I., Sharma, S., Qin, Y.-J., \& Di Matteo, P. 2014, MNRAS, 437, 1284

Raha, N., Sellwood, J. A., James, R. A., \& Kahn, F. D. 1991, Natur, 352,411

Saha, K., Pfenniger, D., \& Taam, R. E. 2013, ApJ, 764, 123

Sellwood, J. A., \& Debattista, V. P. 2009, MNRAS, 398, 1279

Shen, J., \& Li, Z.-Y. 2016, Galactic Bulges, 418, 233

Shen, J., Rich, R. M., Kormendy, J., et al. 2010, ApJL, 720, L72

Sheth, K., Elmegreen, D. M., Elmegreen, B. G., et al. 2008, ApJ, 675, 1141

Simien, F., \& Prugniel, P. 1997, A\&AS, 126, 15

Simien, F., \& Prugniel, P. 2000, A\&AS, 145, 263

Veilleux, S., Bland-Hawthorn, J., \& Cecil, G. 1999, AJ, 118, 2108

Wozniak, H., \& Michel-Dansac, L. 2009, A\&A, 494, 11 